\title{
Investigation of Interactions Between Co-fired LTCC Components
}

\author{
H.Birol, T.Maeder, C.Jacq, P.Ryser \\ EPFL, Laboratoire de Production Microtechnique, CH-1015 Lausanne, Switzerland \\ First presented at Electroceramics IX conference, Cherbourg (FR), 31.5 - 3.6.2004 \\ Version of record : Journal of the European Ceramic Society 25 (12), 2065-2069, 2005. \\ http://hdl.handle.net/10.1016/j.jeurceramsoc.2005.03.182
}

\begin{abstract}
In spite of many advantages it offers for micro-electronic packaging, the low temperature cofired ceramic (LTCC) technology has yet certain issues to be solved. Physical and chemical reactions, for instance, within and between the passive electronic components (thick film conductors, resistors -TFR) and the LTCC tapes (green sheets) during processing are one of the major challenges of this technology. This study aims to better understand and control the nature of such interactions, which have a direct effect on properties. The work is conducted on PTC (positive temperature coefficient) resistors, which are screen printed on (co-fired) and in (buried) LTCC sheets and fired at various temperatures. The final properties such as TCR (temperature coefficient of resistance) and sheet resistance are evaluated in terms of processing parameters using SEM (scanning electron microscopy), dilatometry and EDS (electro dispersive x-ray) analysis as characterization tools. The results show that the TFR properties of buried samples deviate more strongly from the expected values compared to those of co-fired ones. It is primarily related to the destroyed conductor and resistor lines, which is due to the entrapped gases in buried structures as a result of organic burnout of printed inks.
\end{abstract}

Keywords: firing (A), microstructure final (B), electrical properties (C), LTCC

\section{Introduction}

LTCC technology is recently addressed to be a key approach for smart packaging1-5. Although its use initiated in the telecommunication filed due to the excellent dielectric properties of the LTCC tapes, its application areas have diversified in the recent years6-9. Ease of machinability of tapes for 3-D structuration, high production volume and reliability at low processing costs, hermeticity of the circuits have attracted the interest of sensor and mesosystem technologies rapidly. Moreover integration of passive electronic components as screen printable pastes into the tapes and low firing temperatures permitting the use of low resistive terminations have made this technology indispensable for many applications. It is based on LTCC tapes, which sinter below $950^{\circ} \mathrm{C}$ and provide a medium with electrical and mechanical functions for realization of desired products. However the integration of components raises several potentially problematic issues, both physical and chemical. The former arise mainly from the differential shrinkage mismatch of the components and the latter from the chemical interactions of the components during firing. In either case, the extent of 
these effects must be controlled in order to guarantee high reliability. This work aims to better understand the processing-property relationship of the selected TFR's fired on LTCC tapes.

The effects of processing method (co-fired on / in the tapes) and the firing temperature on the TFR properties (TCR and sheet resistance) are studied and the results are interpreted using SEM, dilatometry and compositional analysis.

\section{Experimental Procedure}

The LTCC tape we chose during this study was DuPont (DP) 951-AX ${ }^{10,11}$ which, according to our experience, has well-controlled shrinkage behaviour. On the other hand we used Au-based compositions as terminations (ESL 8837 and DP 5744) and ruthenium-based PTC resistor ink (ESL 2612 with TCR of $2400 \mathrm{ppm} / \mathrm{K}$ and sheet resistance of $100 \mathrm{Ohm}$ at $25^{\circ} \mathrm{C}$ ) due to the reduced deformation obtained on the co-fired tapes by these pastes compared to other systems available at our laboratory.

We applied the conductor-resistor combinations in two variants. In the first one, the pastes were screen-printed on the LTCC sheets and co-fired, whereas in the second one the printed layer was laminated with an additional layer to make a buried structure. Although they were both co-fired, from here on the first set of samples will be referred to as co-fired and the second set as buried for convenience. Screen-printing was made according to the layout shown in figure 1, where the number and the geometry of the resistors are also summarized. After drying the pastes in air and then in a lab oven at $120^{\circ} \mathrm{C}$, the structures were fired according to a two-step firing profile. They were heated up to $440^{\circ} \mathrm{C}$ with a heating rate of $5^{\circ} \mathrm{C} /$ minute ( same rate is applied after the dwell time up to the peak temperature) and kept at that temperature for two hours for organic burnout. This was followed by peak firing temperature of 850,875 and $900^{\circ} \mathrm{C}$ for each structure consecutively for 25 minutes to check the effect of firing temperature on properties. Finally the samples were cooled down to room temperature by a rate of $10^{\circ} \mathrm{C} /$ minute. For each conductor-resistor combination, 4 samples were fired. In order to obtain sufficient statistical interpretation, the resistors with dimensions of $1.5 \mathrm{~mm}: 1.5 \mathrm{~mm}$ were selected since each test pattern has five times of this resistor geometry.

The resistor values were measured three times at 30,65 and $100^{\circ} \mathrm{C}$ between consecutive pad pairs (figure 1), while the temperature was recorded using a Pt-1000 PTC resistor. The measurements were made by Keithley 2000 multimeters and Keithley 7000 scanner using four-wire method. TCR value was calculated based on the resistances at 30 and $100^{\circ} \mathrm{C}$ according to the following relation.

$$
T C R=\left(T_{100}-T_{30}\right) /\left(R_{100}-R_{30}\right) \quad \text { (1) }
$$

where $R$ is the resistance at a certain temperature, $T$. The standard deviation (SD), on the other hand, was calculated by

$$
\mathrm{SD}=\sqrt{\frac{n \sum y^{2}-\left(\sum y\right)}{n(n-1)}}
$$

where $n$ is the number of values $y$ (sheet resistance or TCR). 


\section{Results and Discussions}

\subsection{TCR and Standard Deviation}

TCR and standard deviation data is presented in figure 2.a and b, each figure corresponding to different component pairs and processing variables. Results demonstrate that the co-fired samples have TCR values which are closer to the producer specifications, with much narrower deviation compared to the buried ones. Bearing in mind various effects leading to this result, we started our investigation with the most directly observable one. It was the deformation along the resistor paths in the form of blisters, which formed mechanical distortion on the substrate surface. Although the differential shrinkage is well known for leading to such results it was more dominant in the co-fired samples and not in the buried ones, where the major deformation occurred due to gas evolution as a result of organics burnout and entrapment of these products within the LTCC sheets during firing of the resistor ink. As a result of this, the tape was deformed, severely destroying the thick film paths. Figure 3 shows the SEM analysis of buried components.

On the other hand, the major source of deformation on the co-fired samples was rather different. It was observed that DP 5744 ink created more deformation compared to other composition, which was mainly due to two reasons. The first was related to the differential shrinkage. Dilatometry analysis showed that DP 5744 ink started shrinking from $700^{\circ} \mathrm{C}$ on, where LTCC tape was not dense (figure 4). So the tape basically followed the shrinkage behaviour of the denser layer, which was the paste, until it reached a rigid structure. This was followed by the tape contraction starting from $700^{\circ} \mathrm{C}$, which then balanced the expansion of the ink. The second factor, which contributed to the increased deformation, was related to the nature of the pastes. ESL 8837 ink having a organo metallic character resulted in a very thin fired thickness of around 1 micron, whereas DP 5744 reached a thickness of 6-7 microns (figure 5). It is clearly observed that the paste having higher thickness deformed the tape more than the finer one. In the buried samples, deformation is expected to be much less because the film reside on the neutral plane of the structure and the overall thickness is twice as large.

In addition to these effects influence of the chemical contents of the components on properties was also studied. EDS (electro dispersive $\mathrm{x}$-ray analysis) was performed following XRF (xray fluorescence) and XRD analysis in order to find the elemental compositions. It was found that both the tape and the resistor had common species such as $\mathrm{Pb}, \mathrm{Al}, \mathrm{Ca}$, which are known to be mobile at elevated temperatures. The major chemical interaction, which contributed to highly deviated TCR values of buried samples is believed to be arising from interaction of resistor with the substrate from both sides. Micro-compositional analysis was carried out using EDS and TEM (transmission electron microscopy) to find the chemical composition and its effect on properties will be handled in another paper.

\subsection{Sheet Resistance and Standard Deviation}

Sheet resistance of resistors with $1.5 \mathrm{~mm} \times 1.5 \mathrm{~mm}$ geometry was measured at $30^{\circ} \mathrm{C}$. The results are presented in figure 6.a and b. As it can be seen from the graphs, buried samples showed a higher sheet resistance value with very high standard deviation compared to those of co-fired ones. Among the buried samples, the ones with DP 5744 paste were found to remain closer to the sheet resistance value specified by the producer than the ones with ESL 8837. The origin of this difference is believed to be due to the thickness of the conductor 
paths: as seen in figure 7, ESL 8837 tends to become discontinuous. In buried structures, this is favoured by the interaction with the LTCC glass phase from both sides and also by the mechanical stresses induced by the lamination process.

\section{Conclusion}

Co-firing of LTCC tape with commercial thick film inks at different processing conditions and its effects on properties are studied. It is found that buried samples have increased deviation and unreliable TCR and sheet resistance values compared to the co-fired ones. This is linked to deformation along the resistor paths, which is found to be due to the gases entrapped in LTCC as a result of organics burn-out. Electron microscopy analysis shows that the resistor/conductor lines in these samples were destroyed and partially detached from the substrate. Additionally the introduction of the second LTCC layer acted as an extra diffusion path, which had an influence on properties chemically.

On the other hand the co-fired samples show properties closer to producer specification with lower deviations. It is observed that the thickness of the fired conductor line plays an important role in the extent of deformation in addition to differential shrinkage. This deformation is less in buried structures since the film reside on the neutral plane of the structure and the thickness is twice as large.

\section{References}

1 M.G-Rubio, L.M.S-Laguna, P.J.Moffett and J.J.S-Aviles, The utilization of LTCC-ML Technology for Meso-scale EMS, a Simple Thermistor-based Flow Sensor. Senors and Actuators, 1999, 73, 215-221.

2 M.G-Rubio, L.M.S-Laguna, P.E-Vallejos and J.J.S-Aviles, Overview of LTCC Tape Technology for MesoSystem Technology (MsST). Sensors and Actuators. A, 2001, 89, 222-241.

3 R.R. Tummala, Ceramic and Glass-Ceramic Packaging in the 1990s. J. Am. Ceram. Soc., 1991, 74, 895908.

4 J. Kita, A. Dziedzic, L.J.Golonka and A. Bochenek, Properties of Laser-cut LTCC Heaters. Micro. Rel., 2000, 40, 1005-1010.

5 S. Annas, Advances in Low Temperature Co-Fired Ceramic (LTCC) for Ever Increasing Microelectronic Applications. El. Comp. Tech. Conf., 2003, 1691-1693.

6 L.J.Golonka, B.W. Licznerski, K.Nitsch, H.Teterycz, R.Bauer, K.J. Wolter, Examples of Gas Sensors by Application of Thick film Technology, ,1998, Proc. 43 ${ }^{\text {rd }}$ Int. Sci. Coll., 465-470.

7 A. Dziedzic, L.J.Golonka, J. Kozlowski, W. Benedykt, B.W. Licznerski and K. Nitsch, Thick-film Resistive Temperature Sensors, Meas. Sci. Tech., 1997, 8, 78-85.

8 M.G-Rubio, L.S.-Laguna, M. Smith, and J.J.S.-Aviles, LTCC Technology Multilayer Eddy Current Proximity Sensor for Harsh Environments, 1999, Int. Symp. Microel. , 676-681.

9 H. Lynch, J.Park, P.A.E-Valejos, J.J. S-Aviles, and L.S-Laguna, Meso-scale Pressure Transducers Utilizing LTCC Tapes, 1999, Mat. Res. Soc. Symp. Proc., 546, 177-182.

10 W.K. Jones, Y.Liu, B. Larsen, P. Wang and M. Zampino, Chemical, Structural and Mechanical Properties of LTCC Tapes, 2000, Int. Symp. Microel. , 669-674.

11 A. Dziedzic, L.J. Golonka, J. Kita and J.M. Kozowski, Macro and Microstructure of LTCC Tapes and Components, 2000, IMAPS Poland. 


\begin{tabular}{cccc}
\hline The layout & $\begin{array}{c}\text { Number of } \\
\text { resistors }\end{array}$ & Length & Width \\
\hline & 5 & 1.5 & 1.5 \\
\hline & 1 & 0.3 & 1.5 \\
\hline & 1 & 0.6 & 1.5 \\
\hline & 1 & 1 & 1.5 \\
\hline
\end{tabular}

Fig. 1. The layout for test patterns with description of resistor specifications

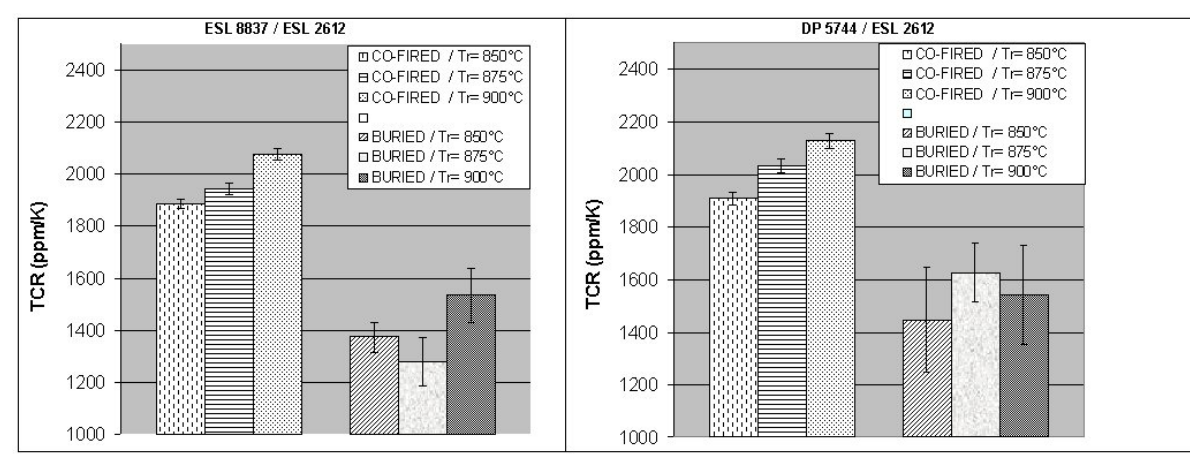

Fig. 2. TCR and standard deviation at different firing conditions for a. ESL 8837- ESL 2612 and b. DP 5744-ESL 2612
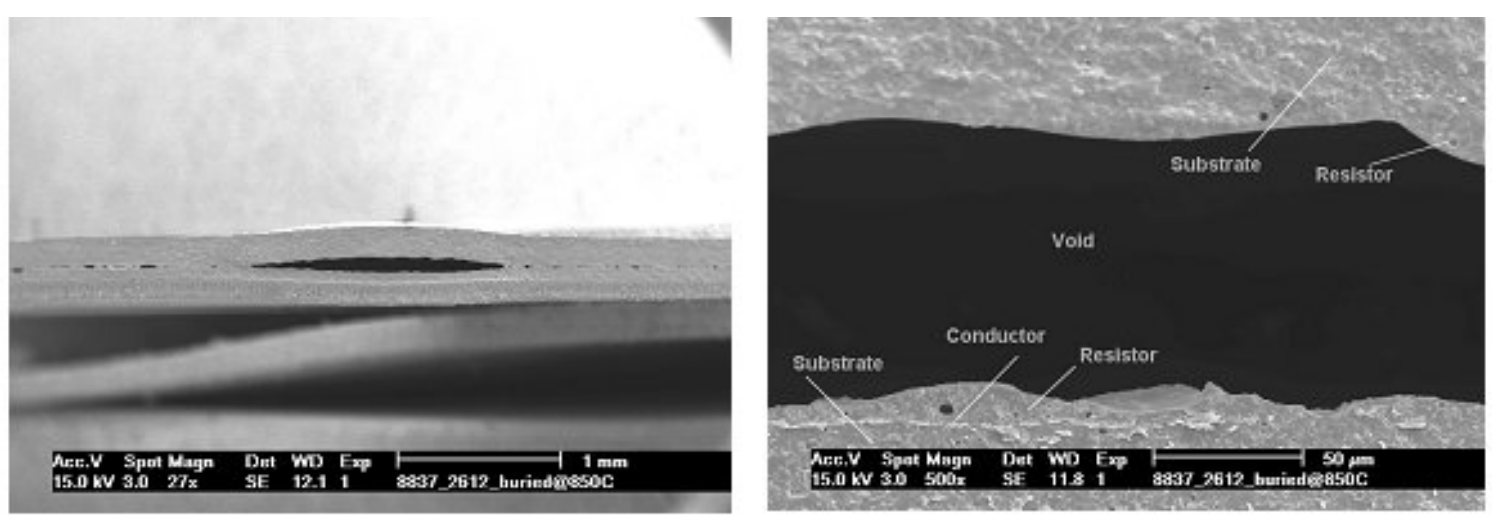

Fig. 3. SEM images of buried ESL 8837 - ESL 2612 component fired at $850^{\circ} \mathrm{C}$. Deformation of the structure due to entrapped gases after organic burn-out. 


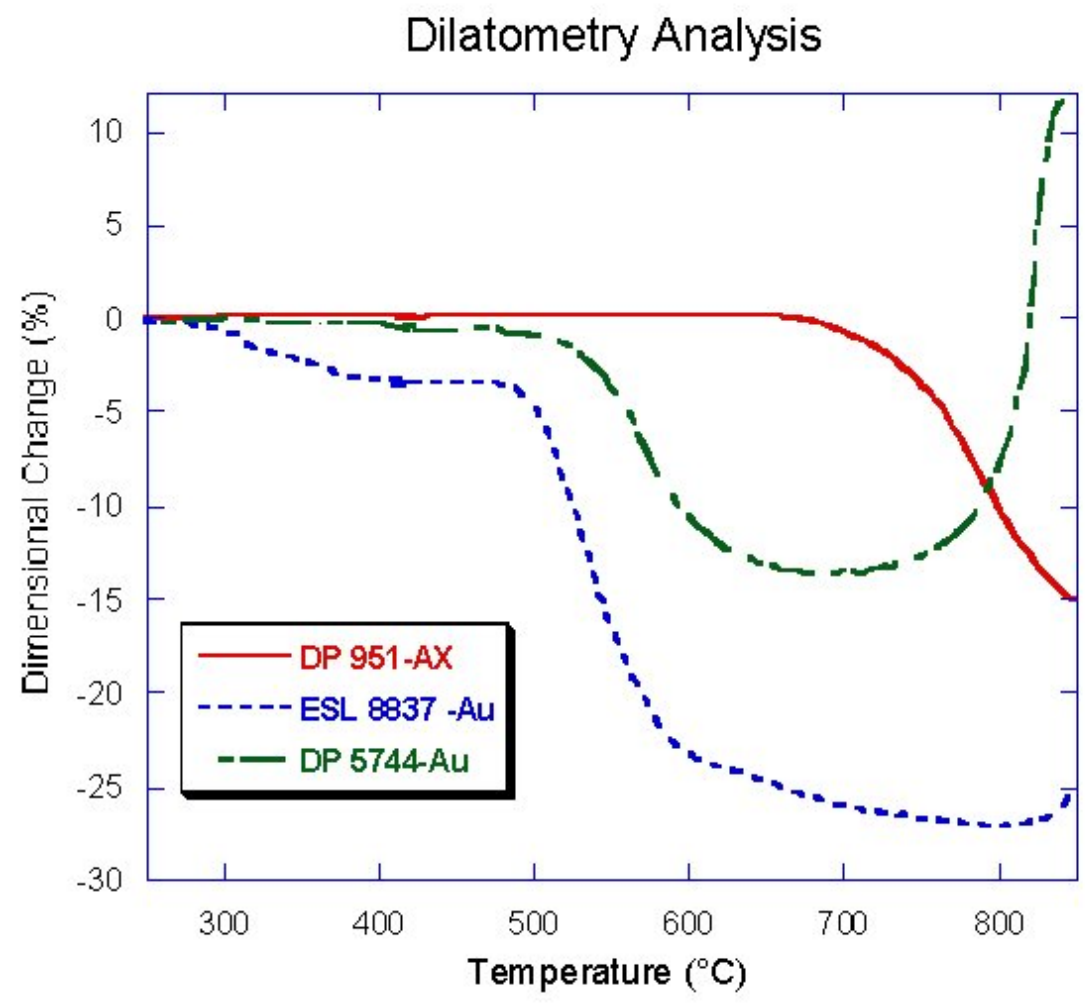

Fig. 4. Dilatometry analysis of the tape and the conductors used. DP 5744-Au ink starts expansion at $700^{\circ} \mathrm{C}$, which is around the onset temperature for the tape shrinkage.
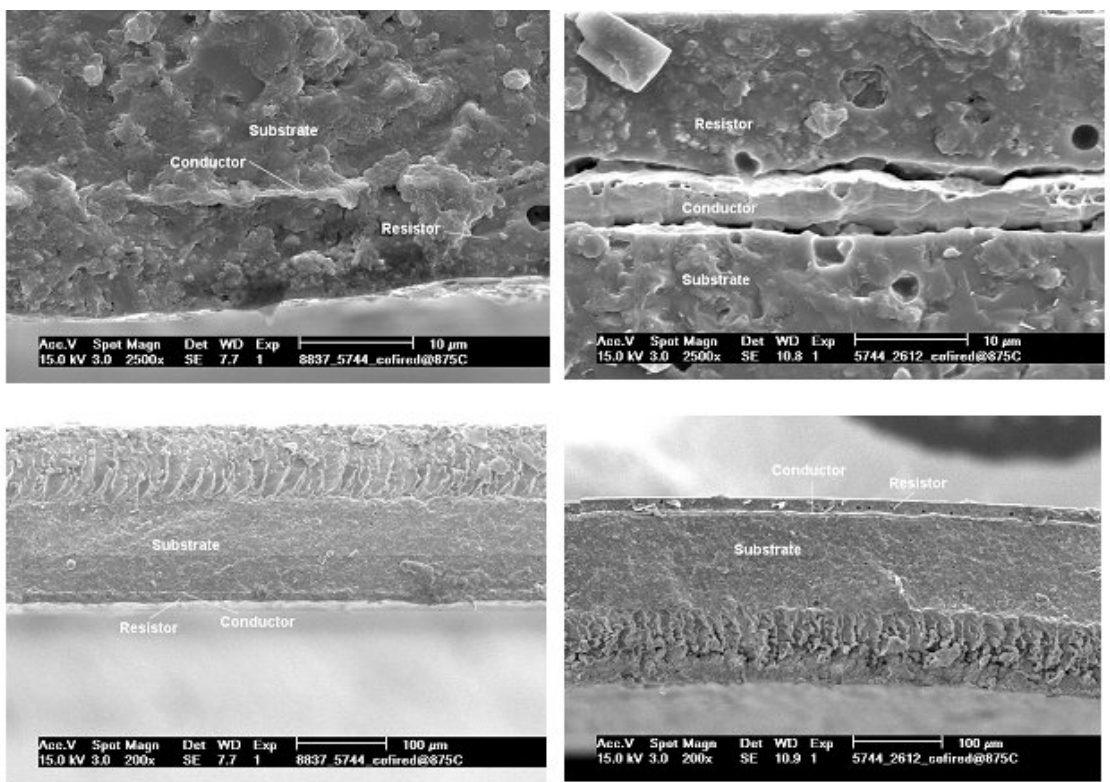

Fig. 5. Comparison of conductor line thickness of ESL 8837 (top left) and DP 5744 at $875^{\circ} \mathrm{C}$ (top right) and its consequence on the substrate deformation. Substrate is curled in the right bottom image (DP 5744). 


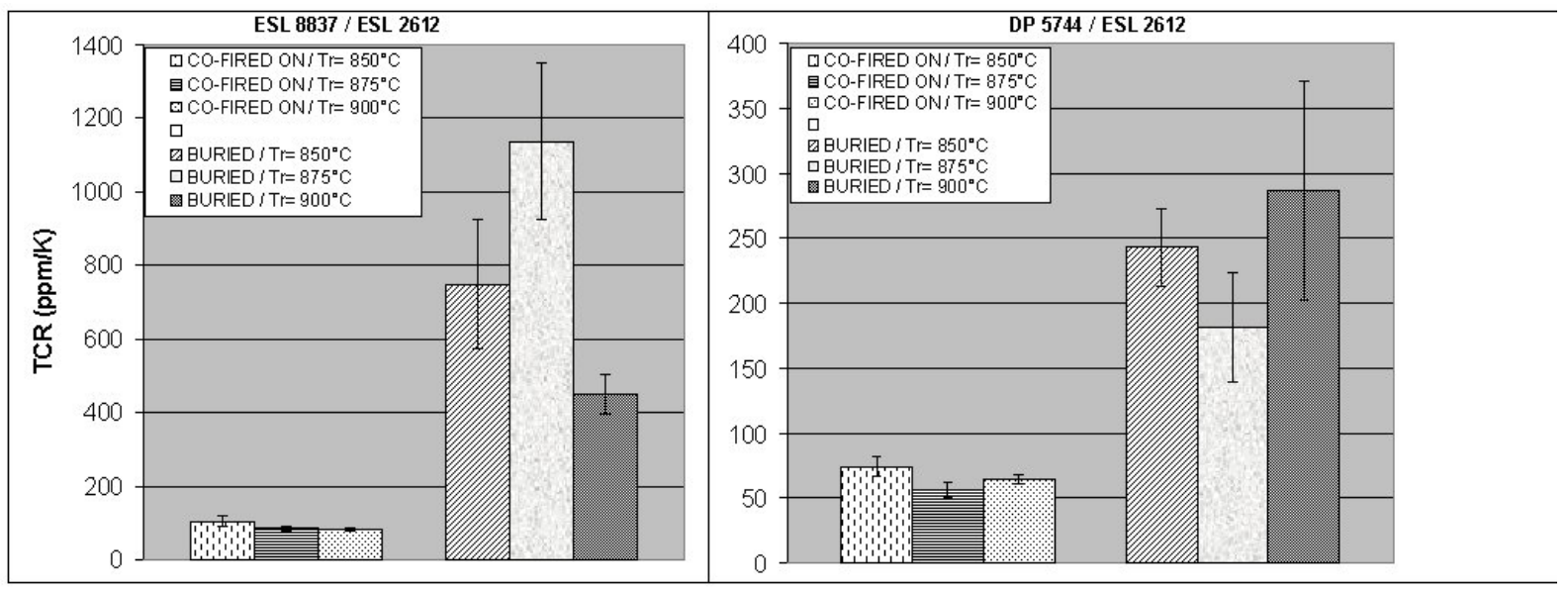

Fig. 6. Sheet resistance and standard deviation at different firing conditions for a. ESL 8837- ESL 2612 and b. DP 5744-ESL 2612

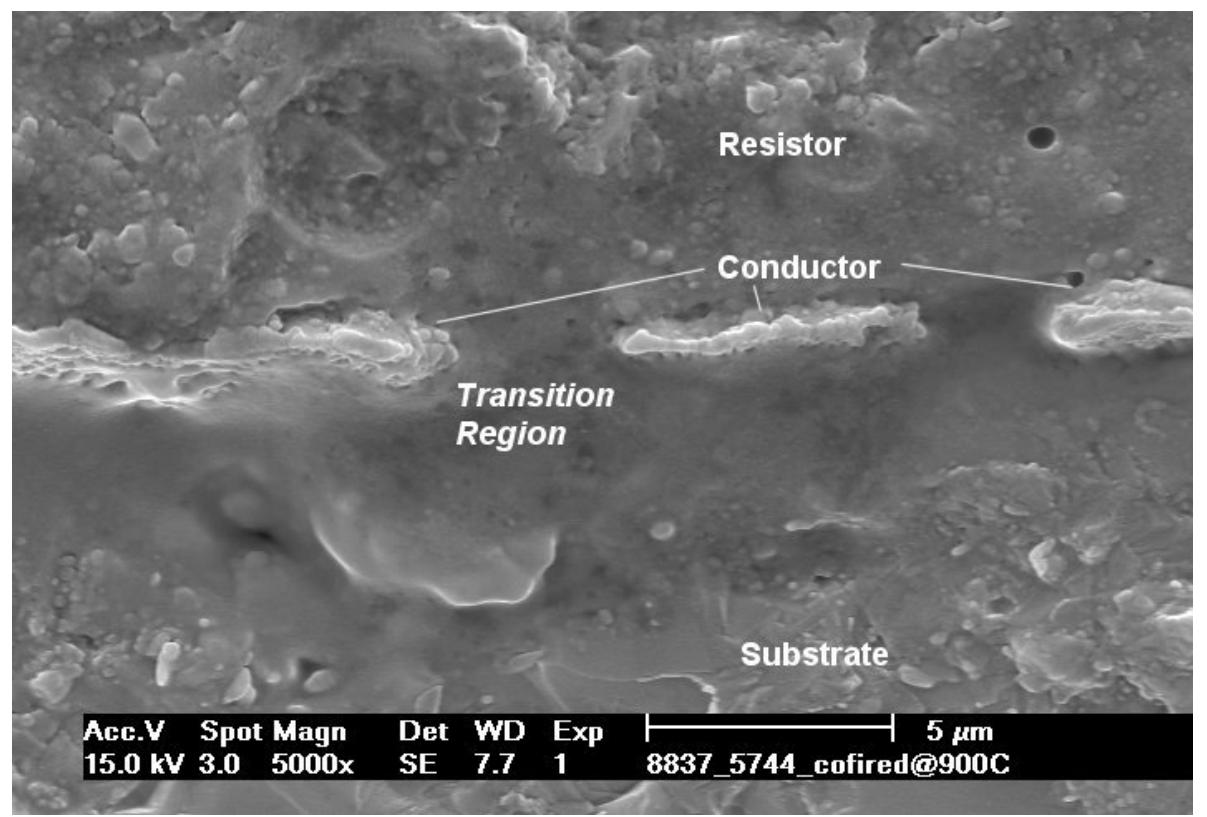

Fig. 7. Discontinuous conductor paths due to glass migration. 